International Journal of Pure and Applied Mathematics

Volume 110 No. 1 2016, 193-209

ISSN: 1311-8080 (printed version); ISSN: 1314-3395 (on-line version)

url: http://www.ijpam.eu

doi: 10.12732/ijpam.v110i1.18

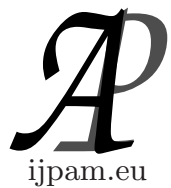

\title{
ITERATIVE ALGORITHM FOR SOLVING THE NEW SYSTEM OF GENERALIZED VARIATIONAL INEQUALITIES IN HILBERT SPACES
}

\author{
Suwicha Imnang ${ }^{1 \S}$, Theeradet Kaewong ${ }^{2}$ and Suthep Suantai ${ }^{3}$ \\ ${ }^{1,2}$ Department of Mathematics and Statistics \\ Faculty of Science \\ Thaksin University \\ Phatthalung, 93210, THAILAND \\ ${ }^{1,2}$ Centre of Excellence in Mathematics, CHE \\ Si Ayutthaya Road, Bangkok 10400, THAILAND \\ ${ }^{3}$ Department of Mathematics \\ Faculty of Science \\ Chiang Mai University \\ Chiang Mai, 50200, THAILAND
}

\begin{abstract}
In this paper, we introduce an iterative method to approximate a common solution of a new general system of variational inequalities, a mixed equilibrium problem and a fixed point problem for a nonexpansive mapping in real Hilbert spaces. We prove that the iterative sequence converges strongly to a common solution of the three problems in the framework of Hilbert spaces. Our main results extend and improve some results in the literature.
\end{abstract}

AMS Subject Classification: 47H10, 49J40, 47H05 47H09, 46B20

Key Words: variational inequality problem, fixed point problem, strong convergence, nonexpansive mapping

\begin{tabular}{lrc}
\hline Received: & February 1, 2016 & \\
Revised: & September 6, 2016 & (C) 2016 Academic Publications, Ltd. \\
Published: & October 21, 2016 & url: www.acadpubl.eu \\
$\S_{\text {Correspondence }}$ author &
\end{tabular}




\section{Introduction}

The study of variational inequality problem is an interesting and fascinating branch of applicable mathematics with a wide range of applications industry, finance, economics, optimization, social, regional, pure and applied science. A closely related subject of current interest is the problem of finding common elements in the fixed point set of nonlinear operators and in the solution set of monotone variational inequalities; see $[1,2,3]$ and the references therein. For the past years, many existence results and iterative algorithms for various variational inequality and variational inclusion problems have been extended and generalized in various directions using and innovative techniques; see $[4,5$, $6,7]$ and the references therein.

Motivated by recent work going in this direction. In this paper, we introduce a new iterative scheme for finding a common element of the set of solutions of a new general system of variational inequalities, the set of solutions of a mixed equilibrium problem and the set of fixed points of a nonexpansive mapping in a real Hilbert space. Furthermore, we prove that the sequence generated by the iterative scheme converges strongly to a common element of those three sets under some control conditions. The results presented in this paper extend and improve the corresponding results of [6] and many others.

\section{Preliminaries}

Let $H$ be a real Hilbert space with inner product $\langle.,$.$\rangle and let C$ be a nonempty closed convex subset of $H$. A mapping $T: C \rightarrow C$ is said to be nonexpansive mapping if $\|T x-T y\| \leq\|x-y\|$ for all $x, y \in C$. The fixed point set of $T$ is denoted by $F(T):=\{x \in C: T x=x\}$. A mapping $A: C \rightarrow H$ is called $\alpha$-inverse-strongly monotone, if there exists a positive real number $\alpha>0$ such that

$$
\langle A x-A y, x-y\rangle \geq \alpha\|A x-A y\|^{2}, \quad \forall x, y \in C .
$$

Let $A_{i}: C \rightarrow H$ for all $i=1,2,3$ be three mappings, then we consider the new general system of variational inequalities of finding $\left(x^{*}, y^{*}, z^{*}\right) \in C \times C \times C$ such that

$$
\left\{\begin{array}{l}
\left\langle\lambda_{1} A_{1} y^{*}+x^{*}-y^{*}, x-x^{*}\right\rangle \geq 0, \quad \forall x \in C, \\
\left\langle\lambda_{2} A_{2} z^{*}+y^{*}-z^{*}, x-y^{*}\right\rangle \geq 0, \quad \forall x \in C, \\
\left\langle\lambda_{3} A_{3} x^{*}+z^{*}-x^{*}, x-z^{*}\right\rangle \geq 0, \quad \forall x \in C,
\end{array}\right.
$$

where $\lambda_{i}>0$ for all $i=1,2,3$.

Some special cases: 
(I) If $A_{3}=0$ and $z^{*}=x^{*}$, then problem (2.1) reduces to find $\left(x^{*}, y^{*}\right) \in C \times C$ such that

$$
\left\{\begin{array}{l}
\left\langle\lambda_{1} A_{1} y^{*}+x^{*}-y^{*}, x-x^{*}\right\rangle \geq 0, \quad \forall x \in C, \\
\left\langle\lambda_{2} A_{2} x^{*}+y^{*}-x^{*}, x-y^{*}\right\rangle \geq 0, \quad \forall x \in C,
\end{array}\right.
$$

which is called a general system of variational inequalities and defined by the authors in [6]. The set of solutions of problem (2.2) denoted by $G V I\left(C, A_{1}, A_{2}\right)$.

(II) If $A_{3}=0, z^{*}=x^{*}$ and $A_{1}=A_{2}:=A$, then problem (2.2) reduces to find $\left(x^{*}, y^{*}\right) \in C \times C$ such that

$$
\begin{cases}\left\langle\lambda_{1} A y^{*}+x^{*}-y^{*}, x-x^{*}\right\rangle \geq 0, & \forall x \in C, \\ \left\langle\lambda_{2} A x^{*}+y^{*}-x^{*}, x-y^{*}\right\rangle \geq 0, & \forall x \in C,\end{cases}
$$

which is called the new system of variational inequalities, and defined by the author in [7].

(III) If $A_{3}=A_{2}=0, z^{*}=y^{*}=x^{*}, A_{1}:=A$ and $\lambda_{1}=1$, then problem (2.3) reduces to find $x^{*} \in C$ such that

$$
\left\langle A x^{*}, x-x^{*}\right\rangle \geq 0, \quad \forall x \in C,
$$

which is called the variational inequality problem.

Let $\varphi: C \rightarrow \mathbb{R} \bigcup\{+\infty\}$ be a proper extended real-valued function and $F$ be a bifunction from $C \times C$ to $\mathbb{R}$, where $\mathbb{R}$ is the set of real numbers. In 2008 , Ceng and Yao [8], introduced the mixed equilibrium problem which is to find $x \in C$ such that

$$
F(x, y)+\varphi(y) \geq \varphi(x), \quad \forall y \in C
$$

The set of solution of problem (2.4) is denoted by $M E P(F, \varphi)$. It is easy to see that $x$ is a solution of problem (2.4) implies that $x \in \operatorname{dom} \varphi=\{x \in C \mid \varphi(x)<$ $+\infty\}$. If $\varphi=0$, then the problem (2.4) reduces to find $x \in C$ such that

$$
F(x, y) \geq 0, \quad \forall y \in C
$$

which is called the equilibrium problem. The set of solution of (2.5) is denoted by $\operatorname{EP}(F)$. In recent yeas, the equilibrium problem has been intensively studied by many authors (see, for example $[1,9,10]$ and references therein).

We recall the well-known results and give some useful lemmas that are used in the next section.

For every point $x \in H$, there exists a unique nearest point in $C$, denoted by $P_{C} x$, such that $\left\|x-P_{C} x\right\| \leq\|x-y\|, \quad \forall y \in C . P_{C}$ is called the metric 
projection of $H$ onto $C$. It is well known that $P_{C}$ is a nonexpansive mapping of $H$ onto $C$ and satisfies

$$
\left\langle x-y, P_{C} x-P_{C} y\right\rangle \geq\left\|P_{C} x-P_{C} y\right\|^{2}, \quad \forall x, y \in H .
$$

Obviously, this immediately implies that

$$
\left\|(x-y)-\left(P_{C} x-P_{C} y\right)\right\|^{2} \leq\|x-y\|^{2}-\left\|P_{C} x-P_{C} y\right\|^{2}, \quad \forall x, y \in H .
$$

Recall that, $P_{C} x$ is characterized by the following properties: $P_{C} x \in C$,

$$
\left\langle x-P_{C} x, y-P_{C} x\right\rangle \leq 0 \text { and }\|x-y\|^{2} \geq\left\|x-P_{C} x\right\|^{2}+\left\|P_{C} x-y\right\|^{2},
$$

for all $x \in H$ and $y \in C$.

For solving the mixed equilibrium problem, let us assume the following assumptions for the bifunction $F, \varphi$ and the set $C$ :

(A1) $F(x, x)=0$ for all $x \in C$;

(A2) $F$ is monotone, i.e. $F(x, y)+F(y, x) \leq 0$ for all $x, y \in C$;

(A3) For each $y \in C, x \mapsto F(x, y)$ is weakly upper semicontinuous;

(A4) For each $x \in C, y \mapsto F(x, y)$ is convex;

(A5) For each $x \in C, y \mapsto F(x, y)$ is lower semicontinuous;

(B1) For each $x \in H$ and $r>0$, there exist a bounded subset $D_{x} \subseteq C$ and $y_{x} \in C$ such that for any $z \in C \backslash D_{x}$,

$$
F\left(z, y_{x}\right)+\varphi\left(y_{x}\right)+\frac{1}{r}\left\langle y_{x}-z, z-x\right\rangle<\varphi(z) .
$$

(B2) $C$ is a bounded set.

In the sequel we shall need to use the following lemma.

Lemma 2.1. ([11]) Let $C$ be a nonempty closed convex subset of $H$. Let $F$ be a bifunction from $C \times C$ to $\mathbb{R}$ satisfying (A1)-(A5) and let $\varphi: C \rightarrow \mathbb{R} \bigcup\{+\infty\}$ be a proper lower semicontinuous and convex function. Assume that either (B1) or (B2) holds. For $r>0$ and $x \in H$, define a mapping $T_{r}: H \rightarrow C$ as follows.

$$
T_{r}(x)=\left\{z \in C: F(z, y)+\varphi(y)+\frac{1}{r}\langle y-z, z-x\rangle \geq \varphi(z), \quad \forall y \in C\right\}
$$

for all $x \in H$. Then the following conclusions hold:

(1) For each $x \in H, T_{r}(x) \neq \varnothing$;

(2) $T_{r}$ is single-valued;

(3) $T_{r}$ is firmly nonexpansive, i.e. for any $x, y \in H$,

$$
\left\|T_{r}(x)-T_{r}(y)\right\|^{2} \leq\left\langle T_{r} x-T_{r} y, x-y\right\rangle ;
$$

(4) $F\left(T_{r}\right)=\operatorname{MEP}(F, \varphi)$;

(5) $\operatorname{MEP}(F, \varphi)$ is closed and convex. 
Lemma 2.2. ([12]) Let $H$ be an inner product space. Then, for all $x, y, z \in H$ and $\alpha, \beta, \gamma \in[0,1]$ with $\alpha+\beta+\gamma=1$, we have

$$
\begin{aligned}
\|\alpha x+\beta y+\gamma z\|^{2}= & \alpha\|x\|^{2}+\beta\|y\|^{2}+\gamma\|z\|^{2}-\alpha \beta\|x-y\|^{2} \\
& -\alpha \gamma\|x-z\|^{2}-\beta \gamma\|y-z\|^{2}
\end{aligned}
$$

Lemma 2.3. In a real Hilbert space $H$, there holds the inequality

$$
\|x+y\|^{2} \leq\|x\|^{2}+2\langle y, x+y\rangle, \quad \forall x, y \in H
$$

Lemma 2.4. ([13]) Assume $\left\{a_{n}\right\}$ is a sequence of nonnegative real numbers such that $a_{n+1} \leq\left(1-\gamma_{n}\right) a_{n}+\delta_{n}$, where $\left\{\gamma_{n}\right\}$ is a sequence in $(0,1)$ and $\left\{\delta_{n}\right\}$ is a sequence such that
(i) $\sum_{n=1}^{\infty} \gamma_{n}=\infty$;
(ii) $\lim \sup _{n \rightarrow \infty} \delta_{n} / \gamma_{n} \leq 0$ or $\sum_{n=1}^{\infty}\left|\delta_{n}\right|<\infty$.

Then $\lim _{n \rightarrow \infty} a_{n}=0$.

Lemma 2.5. ([14]) Let $\left\{x_{n}\right\}$ and $\left\{y_{n}\right\}$ be bounded sequences in a Banach space $X$ and let $\left\{b_{n}\right\}$ be a sequence in $[0,1]$ with $0<\liminf _{n \rightarrow \infty} b_{n} \leq$ $\limsup _{n \rightarrow \infty} b_{n}<1$. Suppose $x_{n+1}=\left(1-b_{n}\right) y_{n}+b_{n} x_{n}$ for all integers $n \geq 1$ and $\lim \sup _{n \rightarrow \infty}\left(\left\|y_{n+1}-y_{n}\right\|-\left\|x_{n+1}-x_{n}\right\|\right) \leq 0$. Then, $\lim _{n \rightarrow \infty}\left\|y_{n}-x_{n}\right\|=0$.

Lemma 2.6. ([15]) Demi-closedness principle. Assume that $T$ is a nonexpansive self-mapping of a nonempty closed convex subset $C$ of a real Hilbert space $H$. If $T$ has a fixed point, then $I-T$ is demi-closed: that is, whenever $\left\{x_{n}\right\}$ is a sequence in $C$ converging weakly to some $x \in C$ (for short, $x_{n} \rightarrow x \in C$ ), and the sequence $\left\{(I-T) x_{n}\right\}$ converges strongly to some $y$ (for short, $\left.(I-T) x_{n} \rightarrow y\right)$, it follows that $(I-T) x=y$. Here $I$ is the identity operator of $H$.

Lemma 2.7. ([16]) Let $C$ be a nonempty closed and convex subset of a real Hilbert space $H$ and $A_{i}: C \rightarrow H$ be three possibly nonlinear mappings, for $i=1,2,3$. Define a mapping $G: C \rightarrow C$ as follows:

$$
\begin{aligned}
G(x)= & P_{C}\left[P_{C}\left(P_{C}\left(x-\lambda_{3} A_{3} x\right)-\lambda_{2} A_{2} P_{C}\left(x-\lambda_{3} A_{3} x\right)\right)\right. \\
& \left.-\lambda_{1} A_{1} P_{C}\left(P_{C}\left(x-\lambda_{3} A_{3} x\right)-\lambda_{2} A_{2} P_{C}\left(x-\lambda_{3} A_{3} x\right)\right)\right], \quad \forall x \in C .
\end{aligned}
$$

For given $x^{*}, y^{*}, z^{*} \in C,\left(x^{*}, y^{*}, z^{*}\right)$ is a solution of problem (2.1) if and only if $x^{*} \in F(G), y^{*}=P_{C}\left(z^{*}-\lambda_{2} A_{2} z^{*}\right)$ and $z^{*}=P_{C}\left(x^{*}-\lambda_{3} A_{3} x^{*}\right)$.

Throughout this paper, the set of fixed points of the mapping $G$ is denoted by $G V I\left(C, A_{1}, A_{2}, A_{3}\right)$. 


\section{Main Results}

In this section, we prove our strong convergence theorem. The next lemma is crucial for proving the main theorem.

Lemma 3.1. Let $C$ be a nonempty closed and convex subset of a real Hilbert space $H$ and let $A_{i}: C \rightarrow H$ be $\alpha_{i}$-inverse-strongly monotone mappings, for $i=1,2,3$. If $\lambda_{i} \in\left(0,2 \alpha_{i}\right]$, for all $i=1,2,3$, then $G: C \rightarrow C$ is nonexpansive, where $G$ is the mapping defined as in Lemma 2.7.

Proof. For all $x, y \in C$, we have

$$
\begin{aligned}
\|G(x)-G(y)\|= & \| P_{C}\left[P_{C}\left(P_{C}\left(I-\lambda_{3} A_{3}\right) x-\lambda_{2} A_{2} P_{C}\left(I-\lambda_{3} A_{3}\right) x\right)\right. \\
& \left.-\lambda_{1} A_{1} P_{C}\left(P_{C}\left(I-\lambda_{3} A_{3}\right) x-\lambda_{2} A_{2} P_{C}\left(I-\lambda_{3} A_{3}\right) x\right)\right] \\
& -P_{C}\left[P_{C}\left(P_{C}\left(I-\lambda_{3} A_{3}\right) y-\lambda_{2} A_{2} P_{C}\left(I-\lambda_{3} A_{3}\right) y\right)\right. \\
& \left.-\lambda_{1} A_{1} P_{C}\left(P_{C}\left(I-\lambda_{3} A_{3}\right) y-\lambda_{2} A_{2} P_{C}\left(I-\lambda_{3} A_{3}\right) y\right)\right] \| \\
\leq & \| P_{C}\left(P_{C}\left(I-\lambda_{3} A_{3}\right) x-\lambda_{2} A_{2} P_{C}\left(I-\lambda_{3} A_{3}\right) x\right) \\
& -\lambda_{1} A_{1} P_{C}\left(P_{C}\left(I-\lambda_{3} A_{3}\right) x-\lambda_{2} A_{2} P_{C}\left(I-\lambda_{3} A_{3}\right) x\right) \\
& -\left[P_{C}\left(P_{C}\left(I-\lambda_{3} A_{3}\right) y-\lambda_{2} A_{2} P_{C}\left(I-\lambda_{3} A_{3}\right) y\right)\right. \\
& \left.-\lambda_{1} A_{1} P_{C}\left(P_{C}\left(I-\lambda_{3} A_{3}\right) y-\lambda_{2} A_{2} P_{C}\left(I-\lambda_{3} A_{3}\right) y\right)\right] \| \\
= & \|\left(I-\lambda_{1} A_{1}\right) P_{C}\left(I-\lambda_{2} A_{2}\right) P_{C}\left(I-\lambda_{3} A_{3}\right) x \\
& -\left(I-\lambda_{1} A_{1}\right) P_{C}\left(I-\lambda_{2} A_{2}\right) P_{C}\left(I-\lambda_{3} A_{3}\right) y \| .
\end{aligned}
$$

It is well known that if $A: C \rightarrow H$ be $\alpha$-inverse-strongly monotone, then $I-\lambda A$ is nonexpansive for all $\lambda \in(0,2 \alpha]$. By our assumption, we obtain $I-\lambda_{i} A_{i}$ is nonexpansive for all $i=1,2,3$. It follows that $\left(I-\lambda_{1} A_{1}\right) P_{C}\left(I-\lambda_{2} A_{2}\right) P_{C}(I-$ $\left.\lambda_{3} A_{3}\right)$ is nonexpansive. Therefore, from (3.1), we obtain immediately that the mapping $G$ is nonexpansive.

Theorem 3.2. Let $C$ be a nonempty closed and convex subset of a real Hilbert space $H$. Let $F$ be a function from $C \times C$ to $\mathbb{R}$ satisfying (A1)$(A 5)$ and $\varphi: C \rightarrow \mathbb{R} \bigcup\{+\infty\}$ be a proper lower semicontinuous and convex function. Let the mappings $A_{i}: C \rightarrow H$ be $\alpha_{i}$-inverse-strongly monotone, for all $i=1,2,3$ and $T$ be a nonexpansive self-mapping of $C$ such that $\Omega=F(T) \bigcap G V I\left(C, A_{1}, A_{2}, A_{3}\right) \bigcap \operatorname{MEP}(F, \varphi) \neq \varnothing$. Assume that either (B1) or (B2) holds and that $v$ is an arbitrary point in $C$. Let $x_{1} \in C$ and 
$\left\{x_{n}\right\},\left\{y_{n}\right\},\left\{z_{n}\right\},\left\{u_{n}\right\}$ be the sequences generated by

$$
\left\{\begin{array}{l}
F\left(u_{n}, y\right)+\varphi(y)-\varphi\left(u_{n}\right)+\frac{1}{r_{n}}\left\langle y-u_{n}, u_{n}-x_{n}\right\rangle \geq 0, \quad \forall y \in C, \\
z_{n}=P_{C}\left(u_{n}-\lambda_{3} A_{3} u_{n}\right), \\
y_{n}=P_{C}\left(z_{n}-\lambda_{2} A_{2} z_{n}\right), \\
x_{n+1}=a_{n} v+b_{n} x_{n}+\left(1-a_{n}-b_{n}\right) T P_{C}\left(y_{n}-\lambda_{1} A_{1} y_{n}\right), \quad n \geq 1,
\end{array}\right.
$$

where $\lambda_{i} \in\left(0,2 \alpha_{i}\right)$, for all $i=1,2,3$ and $\left\{a_{n}\right\},\left\{b_{n}\right\}$ are two sequences in $[0,1]$ and $\left\{r_{n}\right\} \subset(0, \infty)$ satisfying

(C1) $\lim _{n \rightarrow \infty} a_{n}=0$ and $\sum_{n=1}^{\infty} a_{n}=\infty$;

(C2) $0<\liminf _{n \rightarrow \infty} b_{n} \leq \limsup _{n \rightarrow \infty} b_{n}<1$;

(C3) $\liminf _{n \rightarrow \infty} r_{n}>0$ and $\lim _{n \rightarrow \infty}\left|r_{n+1}-r_{n}\right|=0$.

Then $\left\{x_{n}\right\}$ converges strongly to $\bar{x}=P_{\Omega} v$ and $(\bar{x}, \bar{y}, \bar{z})$ is a solution of problem $(2.1)$, where $\bar{y}=P_{C}\left(\bar{z}-\lambda_{2} A_{2} \bar{z}\right)$ and $\bar{z}=P_{C}\left(\bar{x}-\lambda_{3} A_{3} \bar{x}\right)$.

Proof. Step 1. We claim that $\left\{x_{n}\right\}$ is bounded.

Let $x^{*} \in \Omega$ and $\left\{T_{r_{n}}\right\}$ be a sequence of mappings defined as in Lemma 2.1. It follows from Lemma 2.7 that

$$
\begin{aligned}
x^{*}= & P_{C}\left[P_{C}\left(P_{C}\left(x^{*}-\lambda_{3} A_{3} x^{*}\right)-\lambda_{2} A_{2} P_{C}\left(x^{*}-\lambda_{3} A_{3} x^{*}\right)\right)\right. \\
& \left.-\lambda_{1} A_{1} P_{C}\left(P_{C}\left(x^{*}-\lambda_{3} A_{3} x^{*}\right)-\lambda_{2} A_{2} P_{C}\left(x^{*}-\lambda_{3} A_{3} x^{*}\right)\right)\right] .
\end{aligned}
$$

Put $y^{*}=P_{C}\left(z^{*}-\lambda_{2} A_{2} z^{*}\right), z^{*}=P_{C}\left(x^{*}-\lambda_{3} A_{3} x^{*}\right)$ and $t_{n}=P_{C}\left(y_{n}-\lambda_{1} A_{1} y_{n}\right)$. Then $x^{*}=P_{C}\left(y^{*}-\lambda_{1} A_{1} y^{*}\right)$ and

$$
x_{n+1}=a_{n} v+b_{n} x_{n}+\left(1-a_{n}-b_{n}\right) T t_{n} .
$$

By nonexpansiveness of $I-\lambda_{i}(i=1,2,3)$, we have

$$
\begin{aligned}
\left\|t_{n}-x^{*}\right\| & =\left\|P_{C}\left(I-\lambda_{1} A_{1}\right) y_{n}-P_{C}\left(I-\lambda_{1} A_{1}\right) y^{*}\right\| \\
& \leq\left\|y_{n}-y^{*}\right\|=\left\|P_{C}\left(I-\lambda_{2} A_{2}\right) z_{n}-P_{C}\left(I-\lambda_{2} A_{2}\right) z^{*}\right\| \\
& \leq\left\|z_{n}-z^{*}\right\|=\left\|P_{C}\left(I-\lambda_{3} A_{3}\right) u_{n}-P_{C}\left(I-\lambda_{3} A_{3}\right) x^{*}\right\| \\
& \leq\left\|u_{n}-x^{*}\right\|=\left\|T_{r_{n}} x_{n}-T_{r_{n}} x^{*}\right\| \leq\left\|x_{n}-x^{*}\right\|,
\end{aligned}
$$

which implies that

$$
\begin{aligned}
\left\|x_{n+1}-x^{*}\right\| & =\left\|a_{n} v+b_{n} x_{n}+\left(1-a_{n}-b_{n}\right) T t_{n}-x^{*}\right\| \\
& \leq a_{n}\left\|v-x^{*}\right\|+b_{n}\left\|x_{n}-x^{*}\right\|+\left(1-a_{n}-b_{n}\right)\left\|t_{n}-x^{*}\right\| \\
& \leq a_{n}\left\|v-x^{*}\right\|+b_{n}\left\|x_{n}-x^{*}\right\|+\left(1-a_{n}-b_{n}\right)\left\|x_{n}-x^{*}\right\|
\end{aligned}
$$




$$
\leq \max \left\{\left\|v-x^{*}\right\|,\left\|x_{1}-x^{*}\right\|\right\} .
$$

Thus, $\left\{x_{n}\right\}$ is bounded. Consequently, the sequences $\left\{y_{n}\right\},\left\{z_{n}\right\},\left\{t_{n}\right\},\left\{A_{1} y_{n}\right\}$, $\left\{A_{2} z_{n}\right\},\left\{A_{3} u_{n}\right\}$ and $\left\{T t_{n}\right\}$ are also bounded.

Step 2. We claim that $\left\|x_{n+1}-x_{n}\right\| \rightarrow 0$ as $n \rightarrow \infty$.

By nonexpansiveness of $P_{C}$ and $I-\lambda_{i} A_{i}(i=1,2,3)$, we have

$$
\begin{aligned}
\left\|t_{n+1}-t_{n}\right\| & =\left\|P_{C}\left(y_{n+1}-\lambda_{1} A_{1} y_{n+1}\right)-P_{C}\left(y_{n}-\lambda_{1} A_{1} y_{n}\right)\right\| \leq\left\|y_{n+1}-y_{n}\right\| \\
& =\left\|P_{C}\left(z_{n+1}-\lambda_{2} A_{2} z_{n+1}\right)-P_{C}\left(z_{n}-\lambda_{2} A_{2} z_{n}\right)\right\| \leq\left\|z_{n+1}-z_{n}\right\| \\
& =\left\|P_{C}\left(u_{n+1}-\lambda_{3} A_{3} u_{n+1}\right)-P_{C}\left(u_{n}-\lambda_{3} A_{3} u_{n}\right)\right\| \\
& \leq\left\|u_{n+1}-u_{n}\right\| .
\end{aligned}
$$

On the other hand, from $u_{n}=T_{r_{n}} x_{n} \in \operatorname{dom} \varphi$ and $u_{n+1}=T_{r_{n+1}} x_{n+1} \in \operatorname{dom} \varphi$, we have

$$
F\left(u_{n}, y\right)+\varphi(y)-\varphi\left(u_{n}\right)+\frac{1}{r_{n}}\left\langle y-u_{n}, u_{n}-x_{n}\right\rangle \geq 0, \quad \forall y \in C,
$$

and

$$
F\left(u_{n+1}, y\right)+\varphi(y)-\varphi\left(u_{n+1}\right)+\frac{1}{r_{n+1}}\left\langle y-u_{n+1}, u_{n+1}-x_{n+1}\right\rangle \geq 0, \quad \forall y \in C .
$$

Putting $y=u_{n+1}$ in (3.4) and $y=u_{n}$ in (3.5), we have

$$
F\left(u_{n}, u_{n+1}\right)+\varphi\left(u_{n+1}\right)-\varphi\left(u_{n}\right)+\frac{1}{r_{n}}\left\langle u_{n+1}-u_{n}, u_{n}-x_{n}\right\rangle \geq 0,
$$

and

$$
F\left(u_{n+1}, u_{n}\right)+\varphi\left(u_{n}\right)-\varphi\left(u_{n+1}\right)+\frac{1}{r_{n+1}}\left\langle u_{n}-u_{n+1}, u_{n+1}-x_{n+1}\right\rangle \geq 0 .
$$

From the monotonicity of $F$, we obtain that

$$
\left\langle u_{n+1}-u_{n}, \frac{u_{n}-x_{n}}{r_{n}}-\frac{u_{n+1}-x_{n+1}}{r_{n+1}}\right\rangle \geq 0,
$$

and hence

$$
\left\langle u_{n+1}-u_{n}, u_{n}-u_{n+1}+u_{n+1}-x_{n}-\frac{r_{n}}{r_{n+1}}\left(u_{n+1}-x_{n+1}\right)\right\rangle \geq 0 .
$$

Then, we have

$$
\left\|u_{n+1}-u_{n}\right\|^{2} \leq\left\langle u_{n+1}-u_{n}, x_{n+1}-x_{n}+\left(1-\frac{r_{n}}{r_{n+1}}\right)\left(u_{n+1}-x_{n+1}\right)\right\rangle
$$




$$
\leq\left\|u_{n+1}-u_{n}\right\|\left\{\left\|x_{n+1}-x_{n}\right\|+\left|1-\frac{r_{n}}{r_{n+1}}\right|\left\|u_{n+1}-x_{n+1}\right\|\right\}
$$

and hence

$$
\left\|u_{n+1}-u_{n}\right\| \leq\left\|x_{n+1}-x_{n}\right\|+\frac{1}{r_{n+1}}\left|r_{n+1}-r_{n}\right|\left\|u_{n+1}-x_{n+1}\right\| .
$$

It follows from (3.3) and (3.6) that

$$
\left\|t_{n+1}-t_{n}\right\| \leq\left\|x_{n+1}-x_{n}\right\|+\frac{1}{r_{n+1}}\left|r_{n+1}-r_{n}\right|\left\|u_{n+1}-x_{n+1}\right\| .
$$

Let $x_{n+1}=b_{n} x_{n}+\left(1-b_{n}\right) w_{n}$ for all $n \geq 1$. Then, we obtain

$$
\begin{aligned}
w_{n+1}-w_{n} & =\frac{x_{n+2}-b_{n+1} x_{n+1}}{1-b_{n+1}}-\frac{x_{n+1}-b_{n} x_{n}}{1-b_{n}} \\
& =\frac{a_{n+1} v+\left(1-a_{n+1}-b_{n+1}\right) T t_{n+1}}{1-b_{n+1}}-\frac{a_{n} v+\left(1-a_{n}-b_{n}\right) T t_{n}}{1-b_{n}} \\
& =\frac{a_{n+1}}{1-b_{n+1}}\left(v-T t_{n+1}\right)+\frac{a_{n}}{1-b_{n}}\left(T t_{n}-v\right)+T t_{n+1}-T t_{n} .
\end{aligned}
$$

By (3.7) and (3.8), we have

$$
\begin{aligned}
\left\|w_{n+1}-w_{n}\right\|-\left\|x_{n+1}-x_{n}\right\| \leq & \frac{a_{n+1}}{1-b_{n+1}}\left\|v-T t_{n+1}\right\|+\frac{a_{n}}{1-b_{n}}\left\|T t_{n}-v\right\| \\
& +\left\|t_{n+1}-t_{n}\right\|-\left\|x_{n+1}-x_{n}\right\| \\
\leq & \frac{a_{n+1}}{1-b_{n+1}}\left\|v-T t_{n+1}\right\|+\frac{a_{n}}{1-b_{n}}\left\|T t_{n}-v\right\| \\
& +\frac{1}{r_{n+1}}\left|r_{n+1}-r_{n}\right|\left\|u_{n+1}-x_{n+1}\right\| .
\end{aligned}
$$

This together with $(\mathrm{C} 1)-(\mathrm{C} 3)$, we obtain that

$$
\limsup _{n \rightarrow \infty}\left\|w_{n+1}-w_{n}\right\|-\left\|x_{n+1}-x_{n}\right\| \leq 0 .
$$

Hence, by Lemma 2.5, we get $\left\|x_{n}-w_{n}\right\| \rightarrow 0$ as $n \rightarrow \infty$. Consequently,

$$
\lim _{n \rightarrow \infty}\left\|x_{n+1}-x_{n}\right\|=\lim _{n \rightarrow \infty}\left(1-b_{n}\right)\left\|w_{n}-x_{n}\right\|=0 .
$$

Step 3. We claim that $\left\|T t_{n}-t_{n}\right\| \rightarrow 0$ as $n \rightarrow 0$. Since

$$
x_{n+1}-x_{n}=a_{n}\left(v-x_{n}\right)+\left(1-a_{n}-b_{n}\right)\left(T t_{n}-x_{n}\right),
$$


therefore

$$
\left\|T t_{n}-x_{n}\right\| \rightarrow 0 \text { as } n \rightarrow \infty \text {. }
$$

Next, we prove that $\lim _{n \rightarrow \infty}\left\|x_{n}-u_{n}\right\|=0$. From Lemma 2.1(3), we have

$$
\begin{aligned}
\left\|u_{n}-x^{*}\right\|^{2} & =\left\|T_{r_{n}} x_{n}-T_{r_{n}} x^{*}\right\|^{2} \leq\left\langle T_{r_{n}} x_{n}-T_{r_{n}} x^{*}, x_{n}-x^{*}\right\rangle \\
& =\left\langle u_{n}-x^{*}, x_{n}-x^{*}\right\rangle=\frac{1}{2}\left\{\left\|u_{n}-x^{*}\right\|^{2}+\left\|x_{n}-x^{*}\right\|^{2}-\left\|x_{n}-u_{n}\right\|^{2}\right\} .
\end{aligned}
$$

Hence

$$
\left\|u_{n}-x^{*}\right\|^{2} \leq\left\|x_{n}-x^{*}\right\|^{2}-\left\|x_{n}-u_{n}\right\|^{2} .
$$

From Lemma 2.2, (3.2) and (3.11), we have

$$
\begin{aligned}
\left\|x_{n+1}-x^{*}\right\|^{2} \leq & a_{n}\left\|v-x^{*}\right\|^{2}+b_{n}\left\|x_{n}-x^{*}\right\|^{2}+\left(1-a_{n}-b_{n}\right)\left\|t_{n}-x^{*}\right\|^{2} \\
\leq & a_{n}\left\|v-x^{*}\right\|^{2}+b_{n}\left\|x_{n}-x^{*}\right\|^{2}+\left(1-a_{n}-b_{n}\right)\left\|u_{n}-x^{*}\right\|^{2} \\
\leq & a_{n}\left\|v-x^{*}\right\|^{2}+b_{n}\left\|x_{n}-x^{*}\right\|^{2} \\
& +\left(1-a_{n}-b_{n}\right)\left[\left\|x_{n}-x^{*}\right\|^{2}-\left\|x_{n}-u_{n}\right\|^{2}\right] \\
\leq & a_{n}\left\|v-x^{*}\right\|^{2}+\left\|x_{n}-x^{*}\right\|^{2}-\left(1-a_{n}-b_{n}\right)\left\|x_{n}-u_{n}\right\|^{2} .
\end{aligned}
$$

It follows that

$$
\begin{aligned}
& \left(1-a_{n}-b_{n}\right)\left\|x_{n}-u_{n}\right\|^{2} \leq a_{n}\left\|v-x^{*}\right\|^{2}+\left\|x_{n}-x^{*}\right\|^{2}-\left\|x_{n+1}-x^{*}\right\|^{2} \\
& \leq a_{n}\left\|v-x^{*}\right\|^{2}+\left(\left\|x_{n}-x^{*}\right\|+\left\|x_{n+1}-x^{*}\right\|\right)\left\|x_{n+1}-x_{n}\right\| .
\end{aligned}
$$

From the conditions (C1), (C2) and (3.9), we obtain

$$
\lim _{n \rightarrow \infty}\left\|x_{n}-u_{n}\right\|=0
$$

By (3.10) and (3.12), we have

$$
\left\|T t_{n}-u_{n}\right\| \leq\left\|T t_{n}-x_{n}\right\|+\left\|x_{n}-u_{n}\right\| \rightarrow 0, \text { as } n \rightarrow \infty .
$$

Next, we show that $\left\|A_{1} y_{n}-A_{1} y^{*}\right\| \rightarrow 0,\left\|A_{2} z_{n}-A_{2} z^{*}\right\| \rightarrow 0$ and $\| A_{3} u_{n}-$ $A_{3} x^{*} \| \rightarrow 0$, as $n \rightarrow \infty$.

From (3.2) and $A_{1}$ is $\alpha_{1}$-inverse-strongly monotone mapping, we have

$$
\begin{aligned}
\left\|x_{n+1}-x^{*}\right\|^{2} \leq & a_{n}\left\|v-x^{*}\right\|^{2}+b_{n}\left\|x_{n}-x^{*}\right\|^{2}+\left(1-a_{n}-b_{n}\right)\left\|t_{n}-x^{*}\right\|^{2} \\
= & a_{n}\left\|v-x^{*}\right\|^{2}+b_{n}\left\|x_{n}-x^{*}\right\|^{2} \\
& +\left(1-a_{n}-b_{n}\right)\left\|P_{C}\left(y_{n}-\lambda_{1} A_{1} y_{n}\right)-P_{C}\left(y^{*}-\lambda_{1} A_{1} y^{*}\right)\right\|^{2} \\
\leq & a_{n}\left\|v-x^{*}\right\|^{2}+b_{n}\left\|x_{n}-x^{*}\right\|^{2}
\end{aligned}
$$




$$
\begin{aligned}
& +\left(1-a_{n}-b_{n}\right)\left\|\left(y_{n}-\lambda_{1} A_{1} y_{n}\right)-\left(y^{*}-\lambda_{1} A_{1} y^{*}\right)\right\|^{2} \\
\leq & a_{n}\left\|v-x^{*}\right\|^{2}+b_{n}\left\|x_{n}-x^{*}\right\|^{2} \\
& +\left(1-a_{n}-b_{n}\right)\left[\left\|y_{n}-y^{*}\right\|^{2}+\lambda_{1}\left(\lambda_{1}-2 \alpha_{1}\right)\left\|A_{1} y_{n}-A_{1} y^{*}\right\|^{2}\right] \\
\leq & a_{n}\left\|v-x^{*}\right\|^{2}+\left\|x_{n}-x^{*}\right\|^{2} \\
& +\left(1-a_{n}-b_{n}\right) \lambda_{1}\left(\lambda_{1}-2 \alpha_{1}\right)\left\|A_{1} y_{n}-A_{1} y^{*}\right\|^{2} .
\end{aligned}
$$

Similarly, since $A_{i}$ are $\alpha_{i}$-inverse-strongly monotone mappings for $i=2,3$, $\left\|t_{n}-x^{*}\right\| \leq\left\|y_{n}-y^{*}\right\|$ and $\left\|y_{n}-y^{*}\right\| \leq\left\|z_{n}-z^{*}\right\|$, we can show that

$$
\begin{aligned}
\left\|x_{n+1}-x^{*}\right\|^{2} \leq & a_{n}\left\|v-x^{*}\right\|^{2}+\left\|x_{n}-x^{*}\right\|^{2} \\
& +\left(1-a_{n}-b_{n}\right) \lambda_{2}\left(\lambda_{2}-2 \alpha_{2}\right)\left\|A_{2} z_{n}-A_{2} z^{*}\right\|^{2}
\end{aligned}
$$

and

$$
\begin{aligned}
\left\|x_{n+1}-x^{*}\right\|^{2} \leq & a_{n}\left\|v-x^{*}\right\|^{2}+\left\|x_{n}-x^{*}\right\|^{2} \\
& +\left(1-a_{n}-b_{n}\right) \lambda_{3}\left(\lambda_{3}-2 \alpha_{3}\right)\left\|A_{3} u_{n}-A_{3} x^{*}\right\|^{2} .
\end{aligned}
$$

From (3.14), (3.15) and (3.16), we have

$$
\begin{aligned}
-\left(1-a_{n}-b_{n}\right) \lambda_{1}\left(\lambda_{1}-2 \alpha_{1}\right) \| A_{1} y_{n}- & A_{1} y^{*}\left\|^{2} \leq a_{n}\right\| v-x^{*} \|^{2} \\
& +\left(\left\|x_{n}-x^{*}\right\|+\left\|x_{n+1}-x^{*}\right\|\right)\left\|x_{n+1}-x_{n}\right\|, \\
-\left(1-a_{n}-b_{n}\right) \lambda_{2}\left(\lambda_{2}-2 \alpha_{2}\right) \| A_{2} z_{n}- & A_{2} z^{*}\left\|^{2} \leq a_{n}\right\| v-x^{*} \|^{2} \\
+\left(\left\|x_{n}-x^{*}\right\|+\left\|x_{n+1}-x^{*}\right\|\right)\left\|x_{n+1}-x_{n}\right\| &
\end{aligned}
$$

and

$$
\begin{aligned}
-\left(1-a_{n}-b_{n}\right) \lambda_{3}\left(\lambda_{3}-2 \alpha_{3}\right) \| A_{3} u_{n}- & A_{3} x^{*}\left\|^{2} \leq a_{n}\right\| v-x^{*} \|^{2} \\
& +\left(\left\|x_{n}-x^{*}\right\|+\left\|x_{n+1}-x^{*}\right\|\right)\left\|x_{n+1}-x_{n}\right\| .
\end{aligned}
$$

This together with (C1), (C2) and (3.9), we obtain that

$$
\lim _{n \rightarrow \infty}\left\|A_{1} y_{n}-A_{1} y^{*}\right\|=\lim _{n \rightarrow \infty}\left\|A_{2} z_{n}-A_{2} z^{*}\right\|=\lim _{n \rightarrow \infty}\left\|A_{3} u_{n}-A_{3} x^{*}\right\|=0 .
$$

Next, we prove that $\left\|T t_{n}-t_{n}\right\| \rightarrow 0$ as $n \rightarrow \infty$. From (2.6), (3.2) and nonexpansiveness of $I-\lambda_{2} A_{2}$ and $I-\lambda_{3} A_{3}$, we get

$$
\begin{aligned}
\left\|y_{n}-y^{*}\right\|^{2} & =\left\|P_{C}\left(z_{n}-\lambda_{2} A_{2} z_{n}\right)-P_{C}\left(z^{*}-\lambda_{2} A_{2} z^{*}\right)\right\|^{2} \\
& \leq\left\langle\left(z_{n}-\lambda_{2} A_{2} z_{n}\right)-\left(z^{*}-\lambda_{2} A_{2} z^{*}\right), y_{n}-y^{*}\right\rangle \\
& =\frac{1}{2}\left[\left\|\left(z_{n}-\lambda_{2} A_{2} z_{n}\right)-\left(z^{*}-\lambda_{2} A_{2} z^{*}\right)\right\|^{2}+\left\|y_{n}-y^{*}\right\|^{2}\right.
\end{aligned}
$$




$$
\begin{aligned}
& \left.-\left\|\left(z_{n}-\lambda_{2} A_{2} z_{n}\right)-\left(z^{*}-\lambda_{2} A_{2} z^{*}\right)-\left(y_{n}-y^{*}\right)\right\|^{2}\right] \\
\leq & \frac{1}{2}\left[\left\|z_{n}-z^{*}\right\|^{2}+\left\|y_{n}-y^{*}\right\|^{2}\right. \\
& \left.-\left\|\left(z_{n}-y_{n}\right)-\left(z^{*}-y^{*}\right)-\lambda_{2}\left(A_{2} z_{n}-A_{2} z^{*}\right)\right\|^{2}\right] \\
\leq & \frac{1}{2}\left[\left\|x_{n}-x^{*}\right\|^{2}+\left\|y_{n}-y^{*}\right\|^{2}-\left\|\left(z_{n}-y_{n}\right)-\left(z^{*}-y^{*}\right)\right\|^{2}\right. \\
& \left.+2 \lambda_{2}\left\langle\left(z_{n}-y_{n}\right)-\left(z^{*}-y^{*}\right), A_{2} z_{n}-A_{2} z^{*}\right\rangle-\lambda_{2}^{2}\left\|A_{2} z_{n}-A_{2} z^{*}\right\|^{2}\right],
\end{aligned}
$$

and

$$
\begin{aligned}
\left\|z_{n}-z^{*}\right\|^{2}= & \left\|P_{C}\left(u_{n}-\lambda_{3} A_{3} u_{n}\right)-P_{C}\left(x^{*}-\lambda_{3} A_{3} x^{*}\right)\right\|^{2} \\
\leq & \left\langle\left(u_{n}-\lambda_{3} A_{3} u_{n}\right)-\left(x^{*}-\lambda_{3} A_{3} x^{*}\right), z_{n}-z^{*}\right\rangle \\
= & \frac{1}{2}\left[\left\|\left(u_{n}-\lambda_{3} A_{3} u_{n}\right)-\left(x^{*}-\lambda_{3} A_{3} x^{*}\right)\right\|^{2}+\left\|z_{n}-z^{*}\right\|^{2}\right. \\
& \left.-\left\|\left(u_{n}-\lambda_{3} A_{3} u_{n}\right)-\left(x^{*}-\lambda_{3} A_{3} x^{*}\right)-\left(z_{n}-z^{*}\right)\right\|^{2}\right] \\
\leq & \frac{1}{2}\left[\left\|u_{n}-x^{*}\right\|^{2}+\left\|z_{n}-z^{*}\right\|^{2}\right. \\
& \left.-\left\|\left(u_{n}-z_{n}\right)-\left(x^{*}-z^{*}\right)-\lambda_{3}\left(A_{3} u_{n}-A_{3} x^{*}\right)\right\|^{2}\right] \\
\leq & \frac{1}{2}\left[\left\|x_{n}-x^{*}\right\|^{2}+\left\|z_{n}-z^{*}\right\|^{2}-\left\|\left(u_{n}-z_{n}\right)-\left(x^{*}-z^{*}\right)\right\|^{2}\right. \\
& \left.+2 \lambda_{3}\left\langle\left(u_{n}-z_{n}\right)-\left(x^{*}-z^{*}\right), A_{3} u_{n}-A_{3} x^{*}\right\rangle-\lambda_{3}^{2}\left\|A_{3} u_{n}-A_{3} x^{*}\right\|^{2}\right] .
\end{aligned}
$$

Therefore

$$
\begin{aligned}
\left\|y_{n}-y^{*}\right\|^{2} \leq & \left\|x_{n}-x^{*}\right\|^{2}-\left\|\left(z_{n}-y_{n}\right)-\left(z^{*}-y^{*}\right)\right\|^{2} \\
& +2 \lambda_{2}\left\langle\left(z_{n}-y_{n}\right)-\left(z^{*}-y^{*}\right), A_{2} z_{n}-A_{2} z^{*}\right\rangle
\end{aligned}
$$

and

$$
\begin{aligned}
\left\|z_{n}-z^{*}\right\|^{2} \leq & \left\|x_{n}-x^{*}\right\|^{2}-\left\|\left(u_{n}-z_{n}\right)-\left(x^{*}-z^{*}\right)\right\|^{2} \\
& +2 \lambda_{3}\left\langle\left(u_{n}-z_{n}\right)-\left(x^{*}-z^{*}\right), A_{3} u_{n}-A_{3} x^{*}\right\rangle .
\end{aligned}
$$

From (3.18) and (3.19), we have

$$
\begin{aligned}
\left\|x_{n+1}-x^{*}\right\|^{2} \leq & a_{n}\left\|v-x^{*}\right\|^{2}+b_{n}\left\|x_{n}-x^{*}\right\|^{2}+\left(1-a_{n}-b_{n}\right)\left\|y_{n}-y^{*}\right\|^{2} \\
\leq & a_{n}\left\|v-x^{*}\right\|^{2}+b_{n}\left\|x_{n}-x^{*}\right\|^{2} \\
& +\left(1-a_{n}-b_{n}\right)\left[\left\|x_{n}-x^{*}\right\|^{2}-\left\|\left(z_{n}-y_{n}\right)-\left(z^{*}-y^{*}\right)\right\|^{2}\right. \\
& \left.+2 \lambda_{2}\left\langle\left(z_{n}-y_{n}\right)-\left(z^{*}-y^{*}\right), A_{2} z_{n}-A_{2} z^{*}\right\rangle\right] \\
\leq & a_{n}\left\|v-x^{*}\right\|^{2}+\left\|x_{n}-x^{*}\right\|^{2}
\end{aligned}
$$




$$
\begin{aligned}
& -\left(1-a_{n}-b_{n}\right)\left\|\left(z_{n}-y_{n}\right)-\left(z^{*}-y^{*}\right)\right\|^{2} \\
& +\left(1-a_{n}-b_{n}\right) 2 \lambda_{2}\left\|\left(z_{n}-y_{n}\right)-\left(z^{*}-y^{*}\right)\right\|\left\|A_{2} z_{n}-A_{2} z^{*}\right\|
\end{aligned}
$$

and

$$
\begin{aligned}
\left\|x_{n+1}-x^{*}\right\|^{2} \leq & a_{n}\left\|v-x^{*}\right\|^{2}+b_{n}\left\|x_{n}-x^{*}\right\|^{2}+\left(1-a_{n}-b_{n}\right)\left\|z_{n}-z^{*}\right\|^{2} \\
\leq & a_{n}\left\|v-x^{*}\right\|^{2}+b_{n}\left\|x_{n}-x^{*}\right\|^{2} \\
& +\left(1-a_{n}-b_{n}\right)\left[\left\|x_{n}-x^{*}\right\|^{2}-\left\|\left(u_{n}-z_{n}\right)-\left(x^{*}-z^{*}\right)\right\|^{2}\right. \\
& \left.+2 \lambda_{3}\left\langle\left(u_{n}-z_{n}\right)-\left(x^{*}-z^{*}\right), A_{3} u_{n}-A_{3} x^{*}\right\rangle\right] \\
\leq & a_{n}\left\|v-x^{*}\right\|^{2}+\left\|x_{n}-x^{*}\right\|^{2} \\
& -\left(1-a_{n}-b_{n}\right)\left\|\left(u_{n}-z_{n}\right)-\left(x^{*}-z^{*}\right)\right\|^{2} \\
& +\left(1-a_{n}-b_{n}\right) 2 \lambda_{3}\left\|\left(u_{n}-z_{n}\right)-\left(x^{*}-z^{*}\right)\right\|\left\|A_{3} u_{n}-A_{3} x^{*}\right\| .
\end{aligned}
$$

Hence

$$
\begin{aligned}
& \left(1-a_{n}-b_{n}\right)\left\|\left(z_{n}-y_{n}\right)-\left(z^{*}-y^{*}\right)\right\|^{2} \\
& \leq a_{n}\left\|v-x^{*}\right\|^{2}+\left(1-a_{n}-b_{n}\right) 2 \lambda_{2}\left\|\left(z_{n}-y_{n}\right)-\left(z^{*}-y^{*}\right)\right\|\left\|A_{2} z_{n}-A_{2} z^{*}\right\| \\
& \quad+\left(\left\|x_{n}-x^{*}\right\|+\left\|x_{n+1}-x^{*}\right\|\right)\left\|x_{n+1}-x_{n}\right\|
\end{aligned}
$$

and

$$
\begin{aligned}
& \left(1-a_{n}-b_{n}\right)\left\|\left(u_{n}-z_{n}\right)-\left(x^{*}-z^{*}\right)\right\|^{2} \\
& \leq a_{n}\left\|v-x^{*}\right\|^{2}+\left(1-a_{n}-b_{n}\right) 2 \lambda_{3}\left\|\left(u_{n}-z_{n}\right)-\left(x^{*}-z^{*}\right)\right\|\left\|A_{3} u_{n}-A_{3} x^{*}\right\| \\
& \quad+\left(\left\|x_{n}-x^{*}\right\|+\left\|x_{n+1}-x^{*}\right\|\right)\left\|x_{n+1}-x_{n}\right\| .
\end{aligned}
$$

This together with (C1), (C2), (3.9) and (3.17), we obtain

$$
\lim _{n \rightarrow \infty}\left\|\left(z_{n}-y_{n}\right)-\left(z^{*}-y^{*}\right)\right\|=\lim _{n \rightarrow \infty}\left\|\left(u_{n}-z_{n}\right)-\left(x^{*}-z^{*}\right)\right\|=0 .
$$

Therefore

$$
\begin{aligned}
\left\|\left(u_{n}-y_{n}\right)-\left(x^{*}-y^{*}\right)\right\| \leq & \left\|\left(z_{n}-y_{n}\right)-\left(z^{*}-y^{*}\right)\right\| \\
& +\left\|\left(u_{n}-z_{n}\right)-\left(x^{*}-z^{*}\right)\right\| \rightarrow 0 \text { as } n \rightarrow \infty .
\end{aligned}
$$

From Lemma 2.3 and (2.7), it follows that

$$
\begin{aligned}
& \left\|\left(y_{n}-t_{n}\right)+\left(x^{*}-y^{*}\right)\right\|^{2}=\|\left(y_{n}-\lambda_{1} A_{1} y_{n}\right)-\left(y^{*}-\lambda_{1} A_{1} y^{*}\right) \\
& \quad-\left[P_{C}\left(y_{n}-\lambda_{1} A_{1} y_{n}\right)-P_{C}\left(y^{*}-\lambda_{1} A_{1} y^{*}\right)\right]+\lambda_{1}\left(A_{1} y_{n}-A_{1} y^{*}\right) \|^{2}
\end{aligned}
$$




$$
\begin{aligned}
\leq & \left\|\left(y_{n}-\lambda_{1} A_{1} y_{n}\right)-\left(y^{*}-\lambda_{1} A_{1} y^{*}\right)-\left[P_{C}\left(y_{n}-\lambda_{1} A_{1} y_{n}\right)-P_{C}\left(y^{*}-\lambda_{1} A_{1} y^{*}\right)\right]\right\|^{2} \\
& +2 \lambda_{1}\left\langle A_{1} y_{n}-A_{1} y^{*},\left(y_{n}-t_{n}\right)+\left(x^{*}-y^{*}\right)\right\rangle \\
\leq & \left\|\left(y_{n}-\lambda_{1} A_{1} y_{n}\right)-\left(y^{*}-\lambda_{1} A_{1} y^{*}\right)\right\|^{2}-\left\|P_{C}\left(y_{n}-\lambda_{1} A_{1} y_{n}\right)-P_{C}\left(y^{*}-\lambda_{1} A_{1} y^{*}\right)\right\|^{2} \\
& +2 \lambda_{1}\left\|A_{1} y_{n}-A_{1} y^{*}\right\|\left\|\left(y_{n}-t_{n}\right)+\left(x^{*}-y^{*}\right)\right\| \\
\leq & \left\|\left(y_{n}-\lambda_{1} A_{1} y_{n}\right)-\left(y^{*}-\lambda_{1} A_{1} y^{*}\right)\right\|^{2}-\| T P_{C}\left(y_{n}-\lambda_{1} A_{1} y_{n}\right) \\
& -T P_{C}\left(y^{*}-\lambda_{1} A_{1} y^{*}\right) \|^{2} \\
& +2 \lambda_{1}\left\|A_{1} y_{n}-A_{1} y^{*}\right\|\left\|\left(y_{n}-t_{n}\right)+\left(x^{*}-y^{*}\right)\right\| \\
\leq & \|\left(y_{n}-\lambda_{1} A_{1} y_{n}\right)-\left(y^{*}-\lambda_{1} A_{1} y^{*}\right) \\
& -\left(T t_{n}-x^{*}\right) \|\left[\left\|\left(y_{n}-\lambda_{1} A_{1} y_{n}\right)-\left(y^{*}-\lambda_{1} A_{1} y^{*}\right)\right\|+\left\|T t_{n}-x^{*}\right\|\right] \\
& +2 \lambda_{1}\left\|A_{1} y_{n}-A_{1} y^{*}\right\|\left\|\left(y_{n}-t_{n}\right)+\left(x^{*}-y^{*}\right)\right\| \\
= & \| u_{n}-T t_{n}+x^{*}-y^{*}-\left(u_{n}-y_{n}\right) \\
& -\lambda_{1}\left(A_{1} y_{n}-A_{1} y^{*}\right) \|\left[\left\|\left(y_{n}-\lambda_{1} A_{1} y_{n}\right)-\left(y^{*}-\lambda_{1} A_{1} y^{*}\right)\right\|+\left\|T t_{n}-x^{*}\right\|\right] \\
& +2 \lambda_{1}\left\|A_{1} y_{n}-A_{1} y^{*}\right\|\left\|\left(y_{n}-t_{n}\right)+\left(x^{*}-y^{*}\right)\right\| .
\end{aligned}
$$

This together with (3.13),(3.17) and (3.21), we obtain $\left\|\left(y_{n}-t_{n}\right)+\left(x^{*}-y^{*}\right)\right\| \rightarrow 0$ as $n \rightarrow \infty$. This together with (3.13) and (3.20), we obtain that

$$
\begin{aligned}
\left\|T t_{n}-t_{n}\right\| \leq & \left\|T t_{n}-u_{n}\right\|+\left\|\left(u_{n}-z_{n}\right)-\left(x^{*}-z^{*}\right)\right\|+\left\|\left(z_{n}-y_{n}\right)-\left(z^{*}-y^{*}\right)\right\| \\
& +\left\|\left(y_{n}-t_{n}\right)+\left(x^{*}-y^{*}\right)\right\| \rightarrow 0, \text { as } n \rightarrow \infty .
\end{aligned}
$$

Step 4. We claim that $\lim \sup _{n \rightarrow \infty}\left\langle v-\bar{x}, x_{n}-\bar{x}\right\rangle \leq 0$, where $\bar{x}=P_{\Omega} v$.

Indeed, since $\left\{t_{n}\right\}$ and $\left\{T t_{n}\right\}$ are two bounded sequences in $C$, we can choose a subsequence $\left\{t_{n_{i}}\right\}$ of $\left\{t_{n}\right\}$ such that $t_{n_{i}} \rightarrow z \in C$ and

$$
\limsup _{n \rightarrow \infty}\left\langle v-\bar{x}, T t_{n}-\bar{x}\right\rangle=\lim _{i \rightarrow \infty}\left\langle v-\bar{x}, T t_{n_{i}}-\bar{x}\right\rangle .
$$

Since $\lim _{n \rightarrow \infty}\left\|T t_{n}-t_{n}\right\|=0$, we obtain that $T t_{n_{i}} \rightarrow z$ as $i \rightarrow \infty$.

Next, we show that $z \in \Omega$.

Since $t_{n_{i}} \rightarrow z$ and $\left\|T t_{n}-t_{n}\right\| \rightarrow 0$, we obtain by Lemma 2.6 that $z \in F(T)$.

From (3.22) and (3.10), we obtain

$$
\left\|t_{n}-x_{n}\right\| \leq\left\|T t_{n}-t_{n}\right\|+\left\|T t_{n}-x_{n}\right\| \rightarrow 0, \text { as } n \rightarrow \infty .
$$

Furthermore, by Lemma 3.1, we have $G: C \rightarrow C$ is nonexpansive. Then, we have

$$
\begin{aligned}
& \left\|t_{n}-G\left(t_{n}\right)\right\|=\left\|P_{C}\left(y_{n}-\lambda_{1} A_{1} y_{n}\right)-G\left(t_{n}\right)\right\| \\
& =\left\|P_{C}\left[P_{C}\left(z_{n}-\lambda_{2} A_{2} z_{n}\right)-\lambda_{1} A_{1} P_{C}\left(z_{n}-\lambda_{2} A_{2} z_{n}\right)\right]-G\left(t_{n}\right)\right\|
\end{aligned}
$$




$$
\begin{aligned}
= & \| P_{C}\left[P_{C}\left(P_{C}\left(u_{n}-\lambda_{3} A_{3} u_{n}\right)-\lambda_{2} A_{2} P_{C}\left(u_{n}-\lambda_{3} A_{3} u_{n}\right)\right)\right. \\
& \left.-\lambda_{1} A_{1} P_{C}\left(P_{C}\left(u_{n}-\lambda_{3} A_{3} u_{n}\right)-\lambda_{2} A_{2} P_{C}\left(u_{n}-\lambda_{3} A_{3} u_{n}\right)\right)\right]-G\left(t_{n}\right) \| \\
= & \left\|G\left(u_{n}\right)-G\left(t_{n}\right)\right\| \leq\left\|u_{n}-t_{n}\right\| \\
\leq & \left\|u_{n}-x_{n}\right\|+\left\|x_{n}-t_{n}\right\|,
\end{aligned}
$$

hence $\lim _{n \rightarrow \infty}\left\|t_{n}-G\left(t_{n}\right)\right\|=0$. Again by Lemma 2.6, we have $z \in G V I\left(C, A_{1}\right.$, $\left.A_{2}, A_{3}\right)$.

Since $t_{n_{i}} \rightarrow z$ and $\left\|x_{n}-t_{n}\right\| \rightarrow 0$, we obtain that $x_{n_{i}} \rightarrow z$. From $\left\|u_{n}-x_{n}\right\| \rightarrow$ 0 , we also obtain that $u_{n_{i}} \rightarrow z$. By using the same argument as that in the proof of [11, Theorem 3.1, pp. 1825], we can show that $z \in M E P(F, \varphi)$. Therefore $z \in \Omega$.

On the other hand, it follows from (2.8), (3.10) and $T t_{n_{i}} \rightarrow z$ as $i \rightarrow \infty$ that

$$
\begin{aligned}
\limsup _{n \rightarrow \infty}\left\langle v-\bar{x}, x_{n}-\bar{x}\right\rangle & =\limsup _{n \rightarrow \infty}\left\langle v-\bar{x}, T t_{n}-\bar{x}\right\rangle=\lim _{i \rightarrow \infty}\left\langle v-\bar{x}, T t_{n_{i}}-\bar{x}\right\rangle \\
& =\langle v-\bar{x}, z-\bar{x}\rangle \leq 0
\end{aligned}
$$

Step 5. We claim that $x_{n} \rightarrow \bar{x}$ as $n \rightarrow \infty$.

Since

$$
\begin{aligned}
\left\|x_{n+1}-\bar{x}\right\|^{2}= & \left\langle a_{n} v+b_{n} x_{n}+\left(1-a_{n}-b_{n}\right) T t_{n}-\bar{x}, x_{n+1}-\bar{x}\right\rangle \\
= & a_{n}\left\langle v-\bar{x}, x_{n+1}-\bar{x}\right\rangle+b_{n}\left\langle x_{n}-\bar{x}, x_{n+1}-\bar{x}\right\rangle \\
& +\left(1-a_{n}-b_{n}\right)\left\langle T t_{n}-\bar{x}, x_{n+1}-\bar{x}\right\rangle \\
\leq & a_{n}\left\langle v-\bar{x}, x_{n+1}-\bar{x}\right\rangle+\frac{1}{2} b_{n}\left(\left\|x_{n}-\bar{x}\right\|^{2}+\left\|x_{n+1}-\bar{x}\right\|^{2}\right) \\
& +\frac{1}{2}\left(1-a_{n}-b_{n}\right)\left(\left\|t_{n}-\bar{x}\right\|^{2}+\left\|x_{n+1}-\bar{x}\right\|^{2}\right) \\
\leq & a_{n}\left\langle v-\bar{x}, x_{n+1}-\bar{x}\right\rangle+\frac{1}{2} b_{n}\left(\left\|x_{n}-\bar{x}\right\|^{2}+\left\|x_{n+1}-\bar{x}\right\|^{2}\right) \\
& +\frac{1}{2}\left(1-a_{n}-b_{n}\right)\left(\left\|x_{n}-\bar{x}\right\|^{2}+\left\|x_{n+1}-\bar{x}\right\|^{2}\right) \\
= & a_{n}\left\langle v-\bar{x}, x_{n+1}-\bar{x}\right\rangle+\frac{1}{2}\left(1-a_{n}\right)\left(\left\|x_{n}-\bar{x}\right\|^{2}+\left\|x_{n+1}-\bar{x}\right\|^{2}\right),
\end{aligned}
$$

which implies that

$$
\left\|x_{n+1}-\bar{x}\right\|^{2} \leq\left(1-a_{n}\right)\left\|x_{n}-\bar{x}\right\|^{2}+2 a_{n}\left\langle v-\bar{x}, x_{n+1}-\bar{x}\right\rangle .
$$

This together with $(\mathrm{C} 1)$ and (3.23), we have by Lemma 2.4 that $\left\{x_{n}\right\}$ converges strongly to $\bar{x}$. This completes the proof. 
If $\varphi=0$ in Theorem 3.2, then, we obtain the following result.

Corollary 3.3. Let $C$ be a nonempty closed and convex subset of a real Hilbert space $H$ and $F$ be a function from $C \times C$ to $\mathbb{R}$ satisfying (A1)(A5). Let the mappings $A_{i}: C \rightarrow H$ be $\alpha_{i}$-inverse-strongly monotone, for all $i=1,2,3$ and $T$ be a nonexpansive self-mapping of $C$ such that $\Omega=$ $F(T) \bigcap G V I\left(C, A_{1}, A_{2}, A_{3}\right) \cap E P(F) \neq \varnothing$. Let $v, x_{1} \in C$ and $\left\{x_{n}\right\},\left\{y_{n}\right\},\left\{z_{n}\right\}$ be the sequences generated by

$$
\left\{\begin{array}{l}
F\left(u_{n}, y\right)+\frac{1}{r_{n}}\left\langle y-u_{n}, u_{n}-x_{n}\right\rangle \geq 0, \quad \forall y \in C \\
z_{n}=P_{C}\left(x_{n}-\lambda_{3} A_{3} x_{n}\right) \\
y_{n}=P_{C}\left(z_{n}-\lambda_{2} A_{2} z_{n}\right) \\
x_{n+1}=a_{n} v+b_{n} x_{n}+\left(1-a_{n}-b_{n}\right) T P_{C}\left(y_{n}-\lambda_{1} A_{1} y_{n}\right), \quad n \geq 1
\end{array}\right.
$$

If $\lambda_{i} \in\left(0,2 \alpha_{i}\right)$, for all $i=1,2,3$ and the sequences $\left\{a_{n}\right\},\left\{b_{n}\right\}$ and $\left\{r_{n}\right\}$ are as in Theorem 3.2, then $\left\{x_{n}\right\}$ converges strongly to $\bar{x}=P_{\Omega} v$ and $(\bar{x}, \bar{y}, \bar{z})$ is a solution of problem (2.1), where $\bar{y}=P_{C}\left(\bar{z}-\lambda_{2} A_{2} \bar{z}\right)$ and $\bar{z}=P_{C}\left(\bar{x}-\lambda_{3} A_{3} \bar{x}\right)$.

If $A_{3}=0, \varphi=0, F(x, y)=0$ and $r_{n}=1$ for all $x, y \in C$ and all $n \in \mathbb{N}$ in Theorem 3.2, then $z_{n}=x_{n}$. By Theorem 3.2, we obtain the following result.

Corollary 3.4. [6, Theorem 3.1] Let $C$ be a nonempty closed and convex subset of a real Hilbert space $H$. Let the mappings $A_{1}, A_{2}: C \rightarrow H$ be $\alpha_{1-}$ inverse-strongly monotone and $\alpha_{2}$-inverse-strongly monotone, respectively. Let $T$ be a nonexpansive self-mapping of $C$ such that $\Omega=F(T) \bigcap G V I\left(C, A_{1}, A_{2}\right) \neq$ $\varnothing$. Assume that $v$ is an arbitrary point in $C$. Let $x_{1} \in C$ and $\left\{x_{n}\right\},\left\{y_{n}\right\}$ be the sequences generated by

$$
\left\{\begin{array}{l}
y_{n}=P_{C}\left(x_{n}-\lambda_{2} A_{2} x_{n}\right) \\
x_{n+1}=a_{n} v+b_{n} x_{n}+\left(1-a_{n}-b_{n}\right) T P_{C}\left(y_{n}-\lambda_{1} A_{1} y_{n}\right), \quad n \geq 1
\end{array}\right.
$$

If $\lambda_{1} \in\left(0,2 \alpha_{1}\right), \lambda_{2} \in\left(0,2 \alpha_{2}\right)$ and the sequences $\left\{a_{n}\right\},\left\{b_{n}\right\}$ are as in Theorem 3.2 , then $\left\{x_{n}\right\}$ converges strongly to $\bar{x}=P_{\Omega} v$ and $(\bar{x}, \bar{y})$ is a solution of problem $(2.2)$, where $\bar{y}=P_{C}\left(\bar{x}-\lambda_{2} A_{2} \bar{x}\right)$.

\section{Acknowledgement}

The authors wish to thank the referees and the editor for valuable comments. The first author was supported by the Thailand Research Fund, the Commission on Higher Education and Thaksin University under Grant MRG5680072. The second author was supported by The Thaksin University Research Fund. 


\section{References}

[1] Y. Wang, C. Zhang, Wiener-Hopf equation technique for solving equilibrium problems and variational inequalities and fixed points of a nonexpansive mapping, J. Inequal. Appl. 2014 (2014), Article ID 286.

[2] Q. Yuan, Y. Zhang, Convergence of a regularization algorithm for nonexpansive and monotone operators in Hilbert spaces, Fixed Point Theory Appl. 2014 (2014), Article ID 180.

[3] Z. Yinying, C. Jiantao, W. Yali, Convergence theorem for equilibrium and variational inequality problems and a family of infinitely nonexpansive mappings in Hilbert spaces, J. Appl. Math. 2014 (2014), Article ID 232541.

[4] A. Abdou, B. A. Alamri, Y. J. Cho, Y. Yao, L. J. Zhu, Parallel algoithms for variational inclusions and fixed points with applications, Fixed Point Theory Appl. 2014 (2014), Article ID 174.

[5] M. A. Noor, K. I. Noor, A. G. Khan, Parallel schemes for solving a system of extended general quasi variational inequalities, Appl. Math. Comput. 245 (2014) 566-574.

[6] L. C. Ceng, C. Y. Wang, J. C. Yao, Strong convergence theorems by a relaxed extragradient method for a general system of variational inequalities, Math. Meth. Oper. Res. 67 (2008) 375-390.

[7] R. U. Verma, On a new system of nonlinear variational inequalities and associated iterative algorithms, Math Sci Res Hot-Line 3 (1999) 65-68.

[8] L. C. Ceng, J. C. Yao, A hybrid iterative scheme for mixed equilibrium problems and fixed point problems, J. Comput. App. Math. 214 (2008) 186-201.

[9] Q. Zhang, Strong convergence of a Halpern-type algorithm for common solutions of fixed point and equilibrium problems, J. Inequal. Appl. 2014 (2014), Article ID 313.

[10] S. Suwannaut, A. Kangtunyakarn, Convergence analysis for the equilibrium problems with numerical results, Fixed Point Theory Appl. 2014 (2014), Article ID 167.

[11] J. W. Peng, J. C. Yao, Strong convergence theorems of iterative schemes based on extragradient method for mixed equilibrium problems and fixed point problems, Math. Comput. Model. 49 (2009) 1816-1828.

[12] M. O. Osilike, D. I. Igbokwe, Weak and strong convergence theorems for fixed points of pseudocontractions and solutions of monotone type operator equations, Comput. Math. Appl. 40 (2000) 559-239.

[13] H. K. Xu, Viscosity approximation methods for nonexpansive mappings, J. Math. Anal. Appl. 298 (2004) 279-291.

[14] T. Suzuki, Strong convergence of Krasnoselskii and Manns type sequences for oneparameter nonexpansive semigroups without Bochner integrals, J. Math. Anal. Appl. 305 (2005) 227-239.

[15] K. Goebel, W. A. Kirk, Topics on metric fixed-point theory, Cambridge University Press, Cambridge (1990).

[16] S. Imnang, Hybrid projection algorithm for a new general system of variational inequalities in Hilbert spaces, ISRN Appl. Math. 2012 (2012), Article ID 482869. 
